\title{
Sudden cardiac death
}

Sir,

Drs. A. Myers and H. A. Dewar recently reported 100 cases of sudden cardiac death with coroner's necropsies in Newcastle upon Tyne and stated that the place of mobile coronary care units in preventing these deaths is clearly limited' (British Heart fournal (1975), 37, 1133). We think that this is a laconic dismissal of an important development which is clearly shown to save patients who have experienced sudden cardiac death, a common cardiac event as the authors have pointed out. Perhaps the authors are right concerning mobile coronary care units with inordinate response times in cities without emergency medical systems incorporating lay training in cardiopulmonary resuscitation. However, our experience has certainly been different in a city of equivalent population to that of Newcastle upon Tyne.

Tampa, Florida, has a population of 300000 . The Rescue Division of the Tampa Fire Department has been providing paramedical service with five units since July 1972. Rescue response time is generally 3 to 5 minutes. The paramedics received their advanced training under the auspices of the Hillsborough County Medical Association and have a strong continuing education programme. These paramedics perform basic and advanced cardiopulmonary resuscitation, including defibrillation, telemetry, intubation (both tracheal and oesophageal), establish an intravenous route, and administer medication (both intravenous and intracardiac), as directed by a physician via radio contact. Electrocardiograms are interpreted by the paramedics on the scene as well as by a coronary care nurse at a hospital base station. Patients are transported to area medical facilities for further care by their personal physician.

Sudden cardiac death was defined for this study as a rapid, unexpected deterioration of vital signs in an individual who was going about his normal activities. Successful resuscitation was defined as treatment at the scene, admission and continued treatment at a hospital with eventual discharge.

From July 1974 to July 1975 , resuscitation was attempted on 296 people found in ventricular fibrillation. The number of these fitting the criteria for sudden cardiac death is unknown. However, 34 who fulfilled the criteria for sudden cardiac death were successfully resuscitated. Out of the 296 people, an additional 34 people who did not fulfil the criteria for sudden cardiac death were successfully resuscitated, giving a 23 per cent overall successful resuscitation rate. This is similar to data reported from the mobile coronary care system in Seattle, Washington (Cobb and Alvarez, 1974).

This dramatic increase in survival from fatal cardiac events equals that accomplished by the institution of hospital coronary care units.

Eric E. Harrison, Edward J. Straub, and Brent D. Amey,

The Cardiology Center,

Tampa General Hospital,

Tampa, Florida, USA.

\section{Reference}

Cobb, L. A., and Alvarez, H. (1974). Medic I: the Seattle system for the management of out-of-hospital emergencies. Personal communication.

This letter was shown to Dr. H. A. Dewar and his co-author who reply as follows:

Sir,

The letter from Dr. Harrison and his colleagues has been passed on to us and we accept that the quoted statement in the article by us was laconic. But it was not inaccurate. We entirely agree with them that the results of a mobile coronary care service manned by paramedical personnel such as they describe, and whose counterpart in the U.K. is that pioneered in Brighton by D. A. Chamberlain and his helpers, are superior to those obtained when doctors alone are primarily involved. In Newcastle one of us (H.A.D.) had hoped shortly to supplement the doctor-manned service (which has been operating in the city since 1968) with specially trained ambulance men who ought to reach cases very much more quickly, but this improvement has 
been blocked by the Dept. of Health and Social Security.

Nevertheless in their letter Dr. Harrison and his colleagues show that in Tampa only 23 per cent of the patients with ventricular fibrillation upon whom resuscitation was attempted had a successful outcome. It is surely not inaccurate to describe such a success as 'limited'-however much one may admire it, as we indeed do. To save the 77 out of every 100 who died, a different approach from that of mobile coronary care is needed. It is arguable that the total disappearance of the habit of cigarette smoking for example might save more lives than any coronary care unit whether mobile or stationary.

A. Myers and H. A. Dewar, The Royal Victoria Infirmary, Newcastle upon Tyne.

\section{Erratum}

In the paper 'The Natural History of rheumatic, aortic regurgitation and indications for surgery', which appeared on pp 147-154, line 19, p. 147, column 2 should read-'Fifty-three patients with moderate aortic regurgitation and 67 patients with moderate or severe aortic regurgitation of nonrheumatic aetiology referred during the same period were also excluded from the study'. 